\title{
Clinical features of gallstone impaction at the ampulla of Vater and the effectiveness of endoscopic biliary drainage without papillotomy
}

Authors

Institutions
Yuichi Takano', Masatsugu Nagahama', Naotaka Maruoka', Eiichi Yamamura', Nobuyuki Ohike², Tomoko Norose², Hiroshi Takahashi

${ }^{1}$ Division of Gastroenterology, Department of Internal Medicine, Showa University Fujigaoka Hospital, Yokohama, Kanagawa, Japan

${ }^{2}$ Department of Diagnostic Pathology, Showa University Fujigaoka Hospital, Yokohama, Kanagawa Japan submitted 4. January 2016 accepted after revision 17. May 2016

\section{Bibliography}

Dol http://dx.doi.org/

10.1055/s-0042-109265

Endoscopy International Open

2016; 04: E806-E811

(c) Georg Thieme Verlag KG

Stuttgart · New York

E-ISSN 2196-9736

Corresponding author

Yuichi Takano

1-30 Fujigaoka, Aoba-ku

Yokohama-shi,

Kanagawa 227-8501

Japan

Phone: +81-45-971-1151

yuichitakano1028@yahoo.co.jp
Background and study aims: Gallstone impaction at the ampulla of Vater is a critical condition, and the standard treatment is endoscopic papillotomy. However, the clinical features remain largely unclear, and some patients are reluctant to undergo papillotomy because of a bleeding tendency. The aim of this study was to clarify the clinical features of gallstone impaction at the ampulla of Vater and to examine the effectiveness of endoscopic biliary drainage without papillotomy.

Patients and methods: We retrospectively examined 30 patients who had undergone endoscopic treatment for gallstone impaction at the ampulla of Vater between 2010 and 2015.

Results: According to the severity classification for acute cholangitis in the Tokyo Guidelines (TG13), the condition was mild in 8 patients, moderate in 14 , and severe in only 8 (27\%), despite the stone impaction at the ampulla of Vater. Hyperamylasemia was observed in 18 patients

\section{Introduction}

Gallstone impaction at the ampulla of Vater can cause cholangitis as well as pancreatitis, and urgent stone removal is critical for improving the patient's condition [1-6]. Occasionally, the clinical course is fatal. However, the clinical features of the condition remain largely unclear. The standard treatment is endoscopic papillotomy, but in recent years, some patients have been reluctant to undergo this procedure because of having bleeding tendencies as a result of oral antithrombotic agents, disseminated intravascular coagulation (DIC), hemodialysis, or liver cirrhosis. Some of these factors are reported to increase the risk of post-papillotomy bleeding [7-9]. For patients at risk of hemorrhage, we performed biliary drainage without papillotomy, despite the stone impaction, followed by a second procedure for stone extraction.
(60\%); computed tomography (CT) revealed clear pancreatitis in 5 cases (17\%). Patients were classified into Group A (13 patients who received biliary drainage with papillotomy) and Group B (17 patients receiving biliary drainage without papillotomy). All patients in Group B had 1 or more types of hemorrhage risk. There were no differences between the 2 groups in the success rate of biliary drainage, the rate of elevated serum amylase the following day, or procedure-related complications. Serum amylase levels decreased the following day in all patients, even in Group B (including the 10 patients with hyperamylasemia and the 3 patients with clear pancreatitis on CT). Conclusions: Even with stone impaction at the ampulla of Vater, typical clinical features of cholangitis and pancreatitis are relatively rare. Biliary drainage without papillotomy is acceptable for gallstone impaction at the ampulla of Vater.

The purpose of this study was to clarify the clinical features of gallstone impaction at the ampulla of Vater and to examine the effectiveness of endoscopic biliary drainage without papillotomy.

\section{Patients and methods \\ $\nabla$}

We retrospectively examined the records of 30 patients who had undergone endoscopic treatment for gallstone impaction at the ampulla of Vater at our hospital between 2010 and 2015. Only patients with gallstones were included, with pancreatic stones excluded; and it was an inclusion criterion that the impacted stone at the ampulla of Vater were endoscopically confirmed $(\bullet$ Fig. 1 and $\bullet$ Fig.2). All patients underwent computed tomography (CT) scanning prior to treatment.

Duodenoscopes with a backward viewing angle of $15^{\circ}$ (JF-260V, Olympus Medical Systems Corp, 


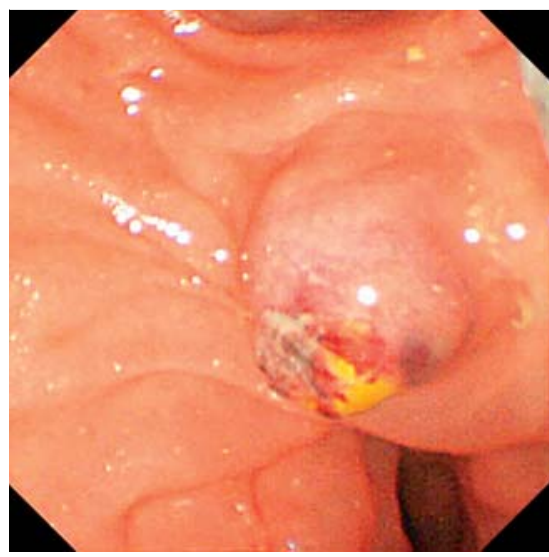

Fig. 1 Gallstone impaction at the orifice of the ampulla of Vater.

Tokyo, Japan) were used in this study. The catheters used were PR-104Q, PR-128Q and PR-V614M (Olympus). The guidewires used were a 0.035-inch straight jagwire (Boston Scientific Japan, Tokyo, Japan) and a 0.025-inch angle visiglide (Olympus). The sphincterotome used was a CleverCut3V (Olympus). The practitioner and assistant were experts who had treated $>500$ patients with endoscopic retrograde cholangiopancreatography (ERCP). The 30 patients were divided according to initial treatment into 2 groups. The 13 patients in Group A had undergone biliary drainage with papillotomy. After biliary cannulation (2 patients required pre-cutting), endoscopic sphincterotomy (EST) was performed with a small-to-medium incision, and a 7-Fr endoscopic nasobiliary drainage (ENBD) tube was installed. The 17 patients in Group B had undergone biliary drainage without papillotomy. All of them had some type of hemorrhage risk. Selective biliary cannulation without pre-cutting was achieved in all patients in Group B. The impacted stone was pushed back into the bile duct using a catheter, and then a 5- or 7-Fr ENBD catheter was put in place $(\bullet$ Video 1$)$.

Repeat ERCP for stone removal was performed in 2 of 13 patients in Group A and in all patients in Group B. The second treatment was performed several days after cholangitis had eased in Group A and after treatment to control the risk of bleeding in Group B. The parameters examined for each patient included 1) clinical features; 2) CT findings; and 3) endoscopic treatment. Statistical analyses were performed using the student's $t$ test and chisquare test to compare the two groups, and probability $(P)$ values of $<0.05$ were considered to indicate a statistically significant difference.

\section{Results}

$\nabla$

\section{Clinical features}

The clinical features of the patients ( 21 male and 9 female) are shown in $\bullet$ Table 1 . The mean \pm standard deviation (SD) age was $76.1 \pm 12.1$ years.

Hyperbilirubinemia (total bilirubin [T-bil] $>2.0 \mathrm{mg} / \mathrm{dL}$ ) was observed in 23 patients (77\%), and hyperamylasemia (serum amylase $[\mathrm{AMY}]>125 \mathrm{U} / \mathrm{L}$ ) in 18 patients $(60 \%)$. According to the severity classification for acute cholangitis in the Tokyo Guidelines (TG13) [10], the condition was mild in 8 patients, moderate in 14 patients, and severe in 8 patients. Twelve patients met the case definition of acute pancreatitis according to the American Collage of Gastroenterology (ACG) guidelines [11] (established by the presence of 2 of the 3 following criteria; (i) abdominal pain consistent with the disease; (ii) serum amylase and/or lipase greater than three times the upper limit of normal; and (iii) characteristic findings from abdominal imaging. According to the revised Atlanta classification, 11 patients had mild pancreatitis and 1 had moderate pancreatitis [12].

Complications included DIC (with a score of $\geq 4$ points according to the scoring system of the Japanese Association for Acute Medicine [13]) in 4 patients and hypotension requiring catecholamines in 4 patients. A total of 12 patients (40\%) received oral antithrombotic agents. One patient had renal failure requiring hemodialysis, 1 had liver cirrhosis because of hepatitis C (ChildPugh class C, 10 points), and 1 had idiopathic thrombocytopenia (ITP). Some patients had multiple hemorrhage risks.

\section{CT findings}

All patients underwent CT scanning prior to treatment ( $\bullet$ Table 2$)$. Five patients $(17 \%)$ had poor common bile duct dilatation $(<10 \mathrm{~mm})$. The stone at the ampulla could be identified in the majority of the patients $(24 / 30 ; 80 \%)$, but there were 6 patients in whom it could not be identified ( Fig. 3 and $\bullet$ Fig.4). Five patients had clear pancreatitis (defined as an elevated concentration of fatty tissue surrounding the pancreas), including 4 patients with inflammation up to the anterior pararenal extraperitoneal space, and 1 patient with inflammation beyond the inferior pole of the kidney.

\section{Endoscopic treatment}

All patients underwent endoscopic treatment. Biliary cannulation was achieved without pre-cutting in 28 patients (93\%), with normal cannulation in 23 of these patients and wire-guided

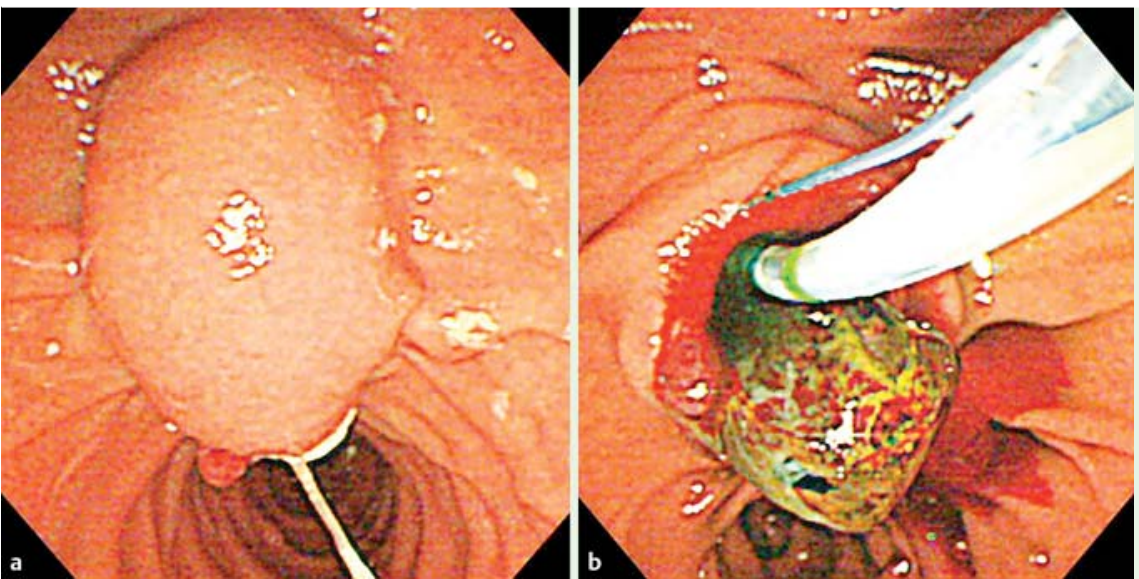

Fig. 2 Although the ampulla of Vater is prominent, the stone cannot be visually confirmed. Gallstone impaction can be diagnosed by papillotomy. The patients in both Fig. 1 and Fig. 2 were diagnosed as gallstone impaction, defined as endoscopically confirmed impacted stone at the ampulla of Vater. 


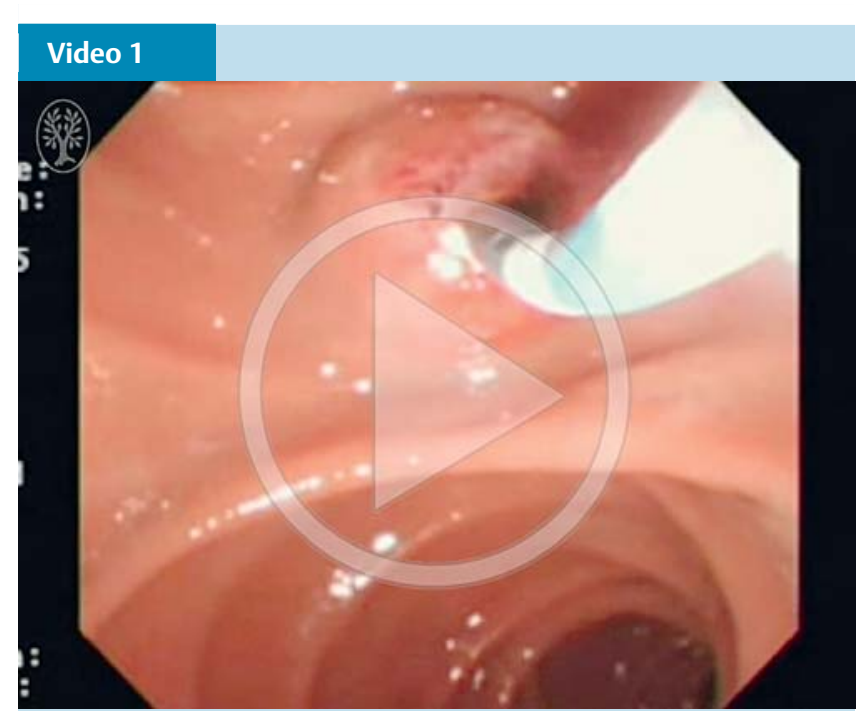

Gallstone impaction can be seen at the ampulla. Deep biliary cannulation was performed to push the stone using the catheter and remove the impaction. Because the patient had multiple hemorrhage risks (oral administration of antithrombotic and hemodialysis), a 7-Fr endoscopic nasobiliary drainage tube was installed without papillotomy. Online content including video sequences viewable at: http://dx.doi.org/10.1055/s-0042-109265

Table 1 Clinical features of impaction cases.

\begin{tabular}{|c|c|}
\hline Age (mean) & $76.1(43 \sim 96)$ \\
\hline Sex & Male 21 : female 9 \\
\hline Hyperbilirubinemia (T-bil>2.0 mg/dL) & $23(77 \%)$ \\
\hline Hyperamylasemia $(A M Y>125 \mathrm{U} / \mathrm{L})$ & $18(60 \%)$ \\
\hline Severity of acute cholangitis ${ }^{1}$ & $\begin{array}{l}\text { Mild } 8(27 \%) \text {, } \\
\text { moderate } 14(46 \%) \text {, } \\
\text { severe } 8(27 \%)\end{array}$ \\
\hline Acute pancreatitis ${ }^{2}$ & $12(40 \%)$ \\
\hline Disseminated intravascular coagulation (DIC) ${ }^{3}$ & $4(13 \%)$ \\
\hline Hypotension requiring catecholamines & $4(13 \%)$ \\
\hline Oral administration of anti-thrombotic agents & $12(40 \%)$ \\
\hline Other bleeding tendencies ${ }^{4}$ & $3(10 \%)$ \\
\hline
\end{tabular}

${ }^{1}$ According to the severity classification for acute cholangitis in the Tokyo Guidelines (TG13).

${ }^{2}$ According to the definition in American Collage of Gastroenterology (ACG) guidelines.

${ }^{3}$ Score of $\geq 4$ points according to the scoring system of the Japanese Association for Acute Medicine.

${ }^{4}$ Hemodialysis in 1, liver cirrhosis in 1, idiopathic thrombocytopenia in 1.

Table 2 CT findings from impaction cases.

\begin{tabular}{|lc|}
\hline Size of common bile duct (mean) & $13.6 \mathrm{~mm}(5.5 \sim 21.1)$ \\
\hline Poor dilatation of common bile duct < $10 \mathrm{~mm}$ & $5(17 \%)$ \\
\hline Size of main pancreatic duct (mean) & $2.3 \mathrm{~mm}(0.9 \sim 6.7)$ \\
\hline Poor dilatation of main pancreatic duct $<2.0 \mathrm{~mm}$ & $17(57 \%)$ \\
\hline Clear pancreatitis ${ }^{1}$ & $5(17 \%)$ \\
\hline Able to identify stone at the ampulla & $24(80 \%)$ \\
\hline
\end{tabular}

${ }^{1}$ Defined as elevated concentration of fatty tissue surrounding the pancreas; 4 patients with inflammation up to the anterior pararenal extraperitoneal space, and 1 patient with inflammation beyond the inferior pole of the kidney. cannulation in the other 5 . In 2 patients, deep cannulation was achieved after pre-cutting (needle knife fistulotomy), and EST was subsequently performed.

The patients in Group A, who underwent biliary drainage with papillotomy, were compared with those in Group B, who underwent biliary drainage without papillotomy. All patients in Group $B$ had some type of hemorrhage risk. No differences were observed between the 2 groups with respect to the other clinical factors ( $\bullet$ Table 3 ).

There were no differences between the 2 groups in the success rate of the biliary drainage, the rate of elevated amylase (AMY) the following day, or procedure-related complications (- Table 4). The serum AMY levels decreased the following day in all patients, even in Group B (including the 10 patients with hyperamylasemia and the 3 patients with clear pancreatitis on CT). Serum AMY level was used as an objective indicator for impaired outflow of pancreatic juice because CT was not repeated, and it was difficult to assess the clinical symptoms precisely. Although there was no statistically significant difference, the duration of treatment was longer in group $A$ than in group $B$.

In Group $A, 11 / 13$ patients underwent complete stone extraction as part of the same procedure. The remaining 2 patients had several stones, and repeat ERCP was performed to remove the stones several days after the inflammation had eased (4 days later for 1 patient and 6 days later for the other). All the patients in group B underwent repeat treatment for stone removal, which was performed at a mean of 6.3 days later (range, 3-14 days). Action was taken to suppress the bleeding tendency as much as possible; antithrombotic agents were stopped temporarily in 6 patients, heparin was replaced in 4 , there was recovery of DIC in 4 , and 1 patient underwent platelet transfusion. Ampulla treatment included endoscopic papillary balloon dilatation (EPBD) in $11 \mathrm{pa}-$ tients and EST in 6 patients. Endoscopic stone extraction was successful in all patients. The hospital stay was significantly longer in group B than in group A.

\section{Discussion \\ $\nabla$}

According to the TG13 classification for the severity of acute cholangitis [10], cholangitis was severe in only 8 (27\%) of the 30 patients, despite the gallstone impaction at the ampulla of Vater. It was mild in 8 patients and moderate in the other 14 patients. The TG13 guidelines also specify the drainage urgency according to the degree of severity ( $\bullet$ Fig. 5) [14]. Urgent drainage is recommended for severe cholangitis, whereas for mild-to-moderate conditions, conservative treatment can be given priority. However, there is a risk of delaying drainage in impaction cases with mild-to-moderate cholangitis; regardless of the severity of the cholangitis, impaction cases need urgent drainage. The pathology of cholangitis involves inflammation of the bile duct (often associated with bacterial infection), and there is a physical obstruction of bile juice flow with stone impaction at the ampulla of Vater. Therefore, even in patients with impaction, some cases present mild-to-moderate cholangitis. This phenomenon is more commonly observed during early onset of impaction.

Hyperamylasemia was observed in 18 patients (60\%), and 5 patients (17\%) exhibited clear pancreatitis as evidenced by imaging. The definition for acute pancreatitis according to the ACG guidelines was satisfied by 12 patients (40\%). There was a lower incidence of pancreatitis than we expected. 

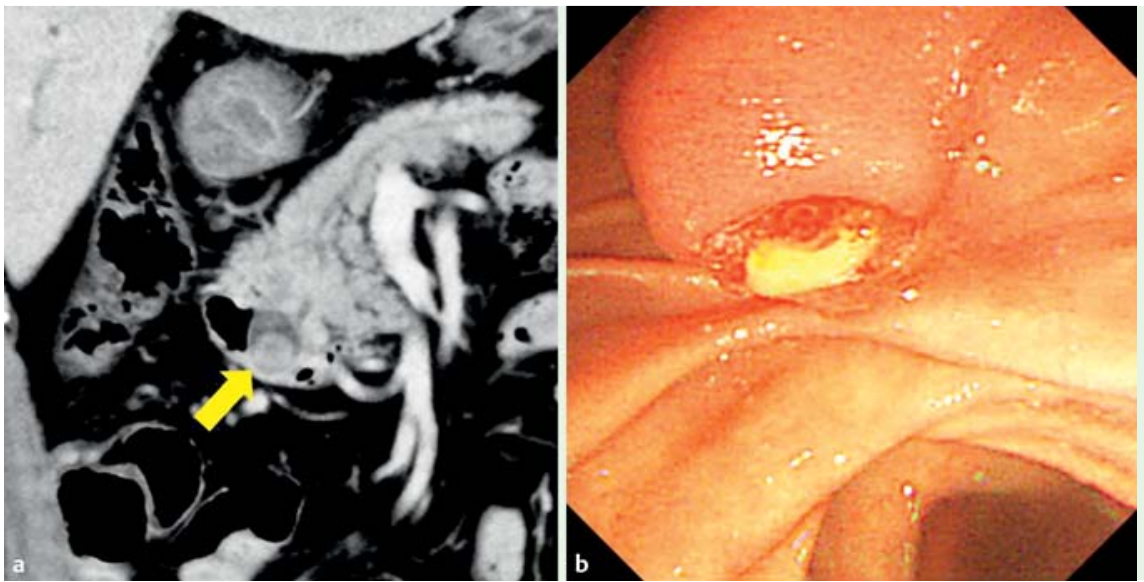

Fig. 3 a Plain CT revealed a high-density stone at the ampulla. $\mathbf{b}$ Stone impaction was found by endoscopy.
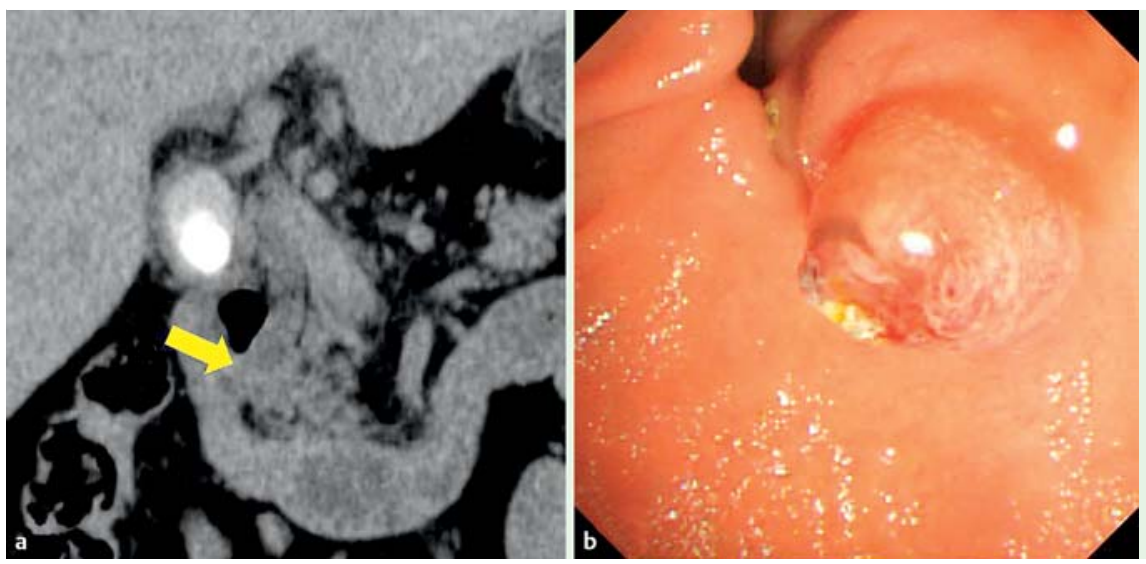

Fig. 4 a A clear stone could not be identified at the ampulla with plain CT. b However, stone impaction was identified with endoscopy. This was an example of a patient for whom identification of the stone was difficult by $\mathrm{CT}$.

Table 3 Comparison of clinical factors between groups.

\begin{tabular}{|c|c|c|c|}
\hline & $\begin{array}{l}\text { Group A: biliary } \\
\text { drainage with } \\
\text { papillotomy }\end{array}$ & $\begin{array}{l}\text { Group B: biliary } \\
\text { drainage without } \\
\text { papillotomy }\end{array}$ & $P$ value \\
\hline Number of patients & 13 & $17^{1}$ & - \\
\hline Age (mean) & 79 & 72 & n.s. ${ }^{2}$ \\
\hline Sex & Male 10 , female 3 & male 11 , female 6 & n. s. ${ }^{3}$ \\
\hline $\begin{array}{l}\text { Severity of acute } \\
\text { cholangitis }\end{array}$ & $\begin{array}{l}\text { Mild } 4 \text {, moderate } \\
6 \text {, severe } 3\end{array}$ & $\begin{array}{l}\text { mild } 4 \text {, moderate } \\
8 \text {, severe } 5\end{array}$ & n.s. ${ }^{3,4}$ \\
\hline $\begin{array}{l}\text { Hyperamylasemia } \\
(\text { AMY }>125 \mathrm{U} / \mathrm{L})\end{array}$ & $8(62 \%)$ & $10(59 \%)$ & n. s. ${ }^{3}$ \\
\hline $\begin{array}{l}\text { Clear pancreatitis } \\
\text { by CT }\end{array}$ & $2(15 \%)$ & $3(18 \%)$ & n. s. ${ }^{3}$ \\
\hline
\end{tabular}

${ }^{1}$ All patients in Group B had some type of hemorrhage risk: oral administration of antithrombotic agents in 12 patients, disseminated intravascular coagulation in 4 patients, hemodialysis in 1 patient, liver cirrhosis in 1 patient, and ITP in 1 patient.

2 Student's $t$ test.

${ }^{3}$ Chi-square test.

${ }^{4}$ Not significant for each grade of cholangitis.

Hyperamylasemia was suggestive of the impaired outflow of pancreatic juice; nevertheless, pancreatitis was not obvious in all the patients on imaging studies.

This may be attributed to the involvement of factors such as the patency of the accessory pancreatic duct, and the degree of impaction. Kamisawa et al. [15] reported that patency of the accessory pancreatic duct with acute pancreatitis was significantly lower than in control cases. They build up a hypothesis that a patent accessory pancreatic duct may function as a second drainage system for the main pancreatic duct, reducing the pressure in the main pancreatic duct and preventing acute pancreatitis. Further-
Table 4 Comparison of endoscopic treatment between two groups.

\begin{tabular}{|llll|}
\hline & $\begin{array}{l}\text { Group A: biliary } \\
\text { drainage with } \\
\text { papillotomy }\end{array}$ & $\begin{array}{l}\text { Group B: biliary } \\
\text { drainage without } \\
\text { papillotomy }\end{array}$ & P value \\
\hline $\begin{array}{l}\text { Successful } \\
\text { biliary drainage }\end{array}$ & $13(100 \%)$ & $17(100 \%)$ & n.s. ${ }^{2}$ \\
\hline $\begin{array}{l}\text { Elevated AMY } \\
\text { levels the } \\
\text { following day }\end{array}$ & $3(23 \%)$ & 0 & n.s. ${ }^{2}$ \\
\hline $\begin{array}{l}\text { Procedure } \\
\text { related } \\
\text { complications }\end{array}$ & $1(8 \%)^{1}$ & 0 & n.s. ${ }^{2}$ \\
\hline $\begin{array}{l}\text { Complete stone } \\
\text { extraction in the } \\
\text { same procedure }\end{array}$ & $11(85 \%)$ & 0 & P<0.00001 \\
\hline $\begin{array}{l}\text { Examination } \\
\text { time(minutes) }\end{array}$ & $26(15-46)$ & $17(9-28)$ & n.s. ${ }^{3}$ \\
\hline $\begin{array}{l}\text { Hospitalization } \\
\text { (Days) }\end{array}$ & $7(4-11)$ & $16(7-29)$ & $P<0.05^{3}$ \\
\hline
\end{tabular}

${ }^{1}$ Minor bleeding

${ }^{2}$ Chi square test.

${ }^{3}$ Student's $t$ test.

more, it may be inferred that the risk of pancreatitis onset differs depending on the degree of impaction. In other words, it is possible that there is a high risk of pancreatitis when severe impaction causing prominent papillary edema and pancreatic duct obstruction is present, whereas the risk of pancreatitis is low when there is mild impaction that only causes biliary obstruction.

In summary, typical clinical features of cholangitis and pancreatitis were rarely associated with gallstone impaction in the current 


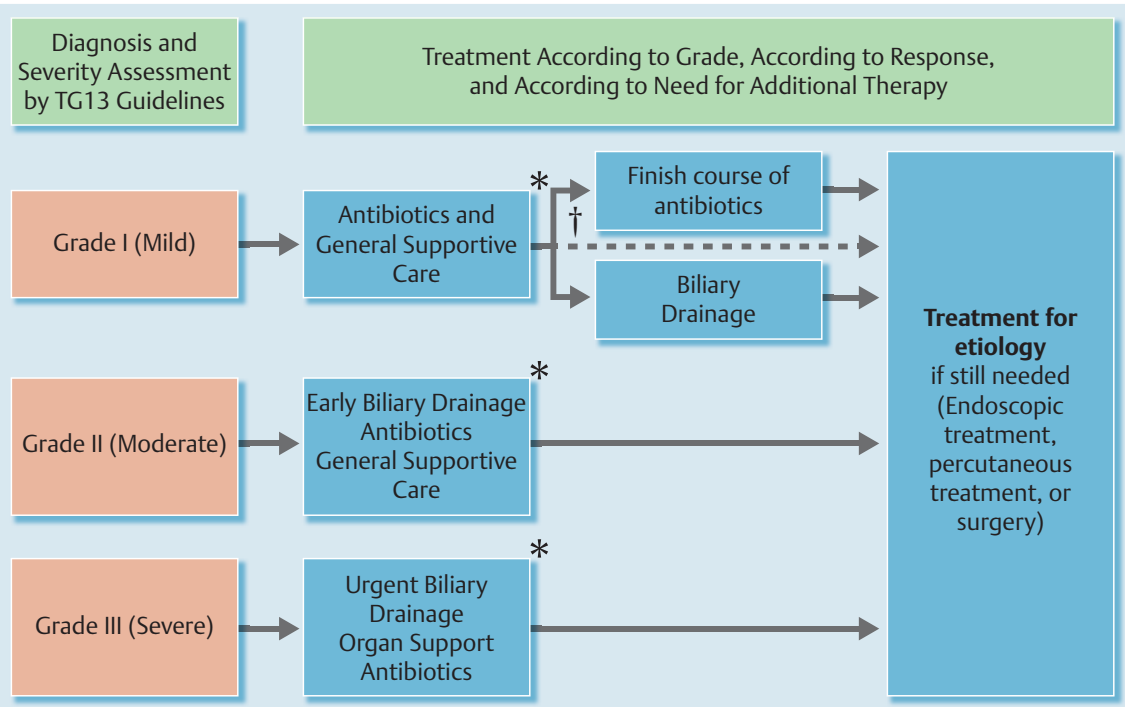

Fig. 5 The recommended treatment course according to the severity of acute cholangitis in the Tokyo Guidelines (TG13). Urgent drainage is recommended in severe cases. For mild and moderate cases, conservative treatment can be given priority.

* Performance of a blood culture should be taken into consideration before initiation of administration of antibiotics. A bile culture should be performed during biliary drainage.

$\dagger$ Principle of treatment for acute cholangitis consists of antimicrobial administration and biliary drainage including treatment for etiology. For patient with choledocholithiasis, treatment for etiology might be performed simultaneously, if possible, with biliary drainage.

study. Kwang et al. examined 46 patients with gallstone impaction at the ampulla of Vater and observed Charcot's triad, the 3 typical findings of cholangitis, in $22 \%$ of the patients, and pancreatitis (defined as abdominal pain plus serum AMY $>3 \times$ upper limit of the normal level) was observed in $37 \%$ [2]. Hence, they reported that typical cholangitis and pancreatitis are rare in patients with impaction, which is similar to our findings.

With impacted stone, cannulation is considered difficult. However, Misra et al. were able to perform normal cannulation in 23/ $32(72 \%)$ patients with impacted stone, reporting that 9 patients required pre-cutting with a needle knife [16]. In our study, we were able to achieve deep cannulation without pre-cutting in $28 / 30$ (93\%) patients. We believe that standard biliary cannulation is possible in most patients, even those with impaction.

Papillotomy is standard treatment for gallstone impaction [1-6]. Dramatic improvements can be expected from the removal of stone. However, post-papillotomy bleeding, which sometimes occurs, can be a problem. Hamada et al. [7] reported that users of anticoagulants are at high risk of bleeding both with EST and with EPBD. Hemodialysis and liver cirrhosis are also reported to increase risks for post-EST bleeding [8,9]. In recent years, many patients have been reluctant to undergo papillotomy because of having a bleeding tendency. For patients at risk of hemorrhage, we perform biliary drainage without papillotomy even for impacted stone, followed by a second procedure for stone extraction. When performing drainage, the impacted stone is pushed back into the bile duct with the catheter. In the current study, serum AMY levels decreased the following day in all patients, even those in Group B. Drainage without papillotomy is acceptable with impacted stone accompanying hyperamylasemia and pancreatitis. In our opinion, the removal of the impacted stone - rather than the papillotomy - is of greatest importance. If the impaction is removed and biliary drainage performed, the patient's condition will improve. In patients at risk of hemorrhage, papillotomy is not required. The best strategy is to prioritize biliary drainage and then perform elective stone extraction. Action should be taken to suppress the bleeding tendency as much as possible.

This treatment strategy, however, has several limitations. First, in a minority of patients, cannulation is not possible without precutting. In this study, 2 patients required pre-cutting for severe impaction and papillary edema. In such patients, at a higher risk of bleeding, pre-cutting had to be given priority. The second limitation is that the hospital stay tended to be longer when only drainage was initially performed because the second procedure for stone extraction was required. An additional limitation is that this retrospective study was conducted at a single facility and included only a small sample population.

In conclusion, the typical clinical features of cholangitis and pancreatitis were rarely associated with gallstone impaction at the ampulla of Vater. The condition of patients will improve with biliary drainage without papillotomy, even those who have hyperamylasemia and pancreatitis. Initial papillotomy is therefore not essential. A treatment strategy that considers the risk of hemorrhage is recommended.

\section{Competing interests: None}

\section{References}

1 Leung JW, Banez VP, Chung SC. Precut (needle knife) papillotomy for impacted common bile duct stone at the ampulla. Am J Gastroenterol 1990; 85: $991-993$

2 Binmoeller KF, Katon RM. Needle knife papillotomy for an impacted common bile duct stone during pregnancy. Gastrointest Endosc 1990; 36: $607-609$

3 Joo KR, Cha JM, Jung SW et al. Case review of impacted bile duct stone at duodenal papilla: detection and endoscopic treatment. Yonsei Med J 2010; 51: 534-539

4 Al-Karawi MA, Ahmed AM, Mohamed AR. Endoscopic choledochoduodenostomy (ECDT): A practical approach to management of impacted papillary gallstones and ampullary tumors. Ann Saudi Med 1992; 12: $352-354$

5 Okabe Y, Kaji R, Ishida $Y$ et al. Successful endoscopic extraction of a large impacted choledocholithiasis in the ampulla of vater: two interesting cases. Dig Endosc 2010; 22: 103 -S106 
6 McAlister VC, Roy A, Passi RB. Harpoon extraction of a common bile duct stone impacted at the ampulla of Vater with needle-knife sphincterotome. Gastrointest Endosc 1993; 39: 111 -112

7 Hamada T, Yasunaga H, Nakai Y et al. Bleeding after endoscopic sphincterotomy or papillary balloon dilation among users of antithrombotic agents. Endoscopy 2015; 47: 997-1004

8 Hori Y, Naitoh I, Nakazawa T et al. Feasibility of endoscopic retrograde cholangiopancreatography-related procedures in hemodialysis patients. J Gastroenterol Hepatol 2014; 29: 648-652

9 Park DH, Kim MH, Lee SK et al. Endoscopic sphincterotomy vs. endoscopic papillary balloon dilation for choledocholithiasis in patients with liver cirrhosis and coagulopathy. Gastrointest Endosc 2004; 60: $180-185$

10 Kiriyama S, Takada T, Strasberg SM et al. TG13 guidelines for diagnosis and severity grading of acute cholangitis (with videos). J Hepatobiliary Pancreat Sci 2013; 20: 24-34
11 Tenner S, Baillie J, DeWitt J et al. American College of Gastroenterology guideline: management of acute pancreatitis. Am J Gastroenterol 2013; 108: 1400 - 1415

12 Banks PA, Bollen TL, Dervenis C et al. Classification of acute pancreatitis - 2012: revision of the Atlanta classification and definitions by international consensus. Gut 2013; 62: $102-111$

13 Gando $S$, Saitoh $D$, Ogura $H$ et al. A multicenter, prospective validation study of the Japanese Association for Acute Medicine disseminated intravascular coagulation scoring system in patients with severe sepsis. Crit Care 2013; 17: R111

14 Miura F, Takada T, Strasberg SM et al. TG13 flowchart for the management of acute cholangitis and cholecystitis. J Hepatobiliary Pancreat Sci 2013; 20: $47-54$

15 Kamisawa T, Takuma K, Tabata $T$ et al. Clinical implications of accessory pancreatic duct. World J Gastroenterol 2010; 16: 4499-4503

16 Misra SP, Dwivedi M. Is conventional sphincterotomy possible in patients with common bile duct stones impacted at the ampulla of Vater? Trop Gastroenterol 2007; 28: 162 - 165 